\title{
Design Study of a 372 GHz Higher Order Mode Input Coupler
}

\author{
Jason R. Garner, Liang Zhang, Craig R. Donaldson, Adrian W. Cross, and Wenlong He
}

\begin{abstract}
The design of a higher order mode (HOM) input coupler for a low-THz gyrotron travelling wave amplifier (gyro-TWA) is presented. A two-branch waveguide coupler based on the even distribution of incident power is designed to couple the rectangular $T_{10}$ mode to the circular $T_{61}$ mode. The optimised tapered waveguide input coupler achieved an operating frequency range of $359-385 \mathrm{GHz}$, equating to a bandwidth of $7 \%$. A prototype waveguide coupler scaled to $\mathrm{W}$-band $(75 \mathrm{GHz}$ to 110GHz) was manufactured. Vector Network Analyser (VNA) measured return loss of the HOM coupler is shown to be $<10 \mathrm{~dB}$ at 90 - 96 GHz. A transmission and phase measurement is presented to analyse $\mathbf{T E}_{61}$ mode conversion in the coupler.
\end{abstract}

Index Terms - Gyro-TWA, mode coupling, waveguide coupler, THz input coupler.

\section{INTRODUCTION}

Gyro-devices are sources of coherent EM radiation based on the cyclotron resonance maser (CRM) instability [1] capable of delivering high power microwave signals at $\mathrm{THz}$ frequencies. Applications of the gyro-devices include plasma heating [2], RADAR systems [3] and spectroscopes [4].

A W-band gyro-TWA has been developed at the University of Strathclyde for use on a cloud profiling RADAR system. The W-band device uses a three-fold helically corrugated interaction region (HCIR) [5]. The HCIR couples the $\mathrm{TE}_{21}$ mode to the first spatial harmonic of the $\mathrm{TE}_{11}$ mode [6] to generate an operating eigenwave, improving the instantaneous bandwidth [7]. A W-band gyrotron backward wave oscillator (gyro-BWO) with similar setup compared to the gyro-TWA achieved a maximum output power of $12 \mathrm{~kW}$ when driven by a $40 \mathrm{kV}, 1.5 \mathrm{~A}$, annular-shaped large-orbit electron beam [8]. The frequency tuning band of $88-102.5 \mathrm{GHz}$ was achieved through cavity magnetic field adjustments.

A gyro-TWA operating at a centre frequency of $372 \mathrm{GHz}$ is being studied for applications of Electron Paramagnetic Resonance (EPR) and Dynamic Nuclear Polarization (DNP) in a Nuclear Magnetic Resonance (NMR) system. High power, wide bandwidth (> 5\%) gyro-amplifiers are ideal for pulsed EPR and DNP-NMR applications. If a 3-fold HCIR is used in the device, the mean radius is $\sim 0.4 \mathrm{~mm}$. Higher order mode (HOM) operation is proposed to increase the cavity dimensions and, hence, enhance the power handling capability of the waveguide cavity.

The gyro-TWA will require efficient coupling of the $\mathrm{TE}_{61}$ mode into the interaction cavity. Minimal power loss at the coupling interface will enable as much of the millimetre wave drive power to be available to the high efficiency interaction.

This work was supported by the Engineering and Physical Sciences Research Council (EPSRC) U.K. under Research Grant EP/K029746/1.

J. R. Garner (j.garner@strath.ac.uk), L. Zhang (liang.zhang@strath.ac.uk), C. R. Donaldson (craig.donaldson@strath.ac.uk), A.W. Cross

(a.w.cross@strath.ac.uk) and W. He (w.he@strath.ac.uk) are with Department of Physics, SUPA, University of Strathclyde Glasgow, G4 0NG, Scotland.
The input coupler was designed focussing on the following three parameters:

1. bandwidth of the input coupler should span the 360 $384 \mathrm{GHz}$ frequency range $(\sim 7 \%)$,

2. transmission loss from $\mathrm{TE}_{10}{ }^{\mathrm{R}}-\mathrm{TE}_{61}{ }^{\mathrm{C}}$ should be less than $1 \mathrm{~dB}$ over the desired frequency range and,

3. mode purity of the $\mathrm{TE}_{61}$ mode should exceed $90 \%$.

By adding the HOM coupling requirement the design of the input coupler is more complex than designing a fundamental mode coupler, due to the possibility of lower order mode (LOM) competition in the cavity. A $\mathrm{TE}_{11}$ field pattern is readily excited and is the dominant mode within a cylindrical waveguide. Therefore, to ensure high $\mathrm{TE}_{61}$ field purity, a complex waveguide coupling geometry is required.

The design of a power dividing input coupler for a $372 \mathrm{GHz}$ HOM gyro-TWA is presented. The numerical modelling of a waveguide splitter input coupler with a linear taper power divider is presented in Section II. The linear taper and E-plane waveguide bend were simulated individually before the full design of the waveguide channel was completed. A tolerance study of the optimised waveguide coupler is discussed in Section III. A prototype waveguide coupler, scaled to operate at $90-96 \mathrm{GHz}$, was manufactured. A discussion of the coupler manufacture is included in Section IV and the Vector Network Analyser prototype testing is presented in Section V. Section VI discusses the design of the coupler at $93 \mathrm{GHz}$ and $372 \mathrm{GHz}$.

\section{II. $372 \mathrm{GHZ}$ TAPERED WAVEGUIDE INPUT COUPLER}

The design of a $372 \mathrm{GHz} \mathrm{TE}_{10}{ }^{\mathrm{R}}-\mathrm{TE}_{61}{ }^{\mathrm{C}}$ waveguide coupler employs the principal of incident wave power distribution to couple to a HOM [9]. The waveguide splitter divides an incident $\mathrm{TE}_{10}$ mode into equal signal intensities, propagating with matched phase towards the cylindrical coupled cavity. The possible modes which may be coupled into the interaction cavity are dependent on the number of branches employed within the designed waveguide splitter. A table of branch numbers and the relevant potential coupled modes, when equal length waveguide branches are used, is shown in Table I. By implementing the waveguide splitter, the generation of a HOM in the interaction cavity is simplified due to a reduction in mode competition.

The design of a waveguide $\mathrm{TE}_{61}{ }^{\mathrm{C}}$ input coupler can include a waveguide splitter with 2 - or 3-branches (Table I). The 3-branch splitter would result in less mode competition inside the cylindrical cavity with a single lower order $\mathrm{TE}_{\mathrm{m} 1}$ mode propagating within the cavity $\left(\mathrm{TE}_{31}\right)$; however, the phase matching of the 3 waveguide channels results in added complexity to the coupler design and manufacture. Therefore, a $\mathrm{TE}_{10}{ }^{\mathrm{R}}-\mathrm{TE}_{61}{ }^{\mathrm{C}}$ input coupler with a 2-branch waveguide splitter was designed for a $360-384 \mathrm{GHz}$ gyro-TWA.

A tapered waveguide can be employed to provide an impedance match between two waveguides of similar geometries without the complex manufacturing of step discontinuities. Tapered waveguides have been designed with 


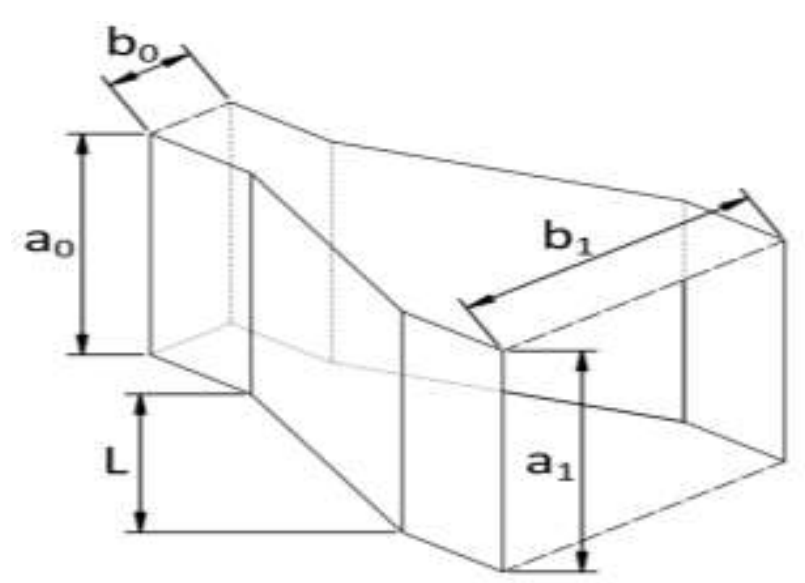

Fig. 1: Schematic of a rectangular-to-square linearly tapered waveguide. linear or part-sinusoidal axial tapers with single E-plane variations [10], or double tapers which vary in the E- and $\mathrm{H}$ plane simultaneously [11]. A linearly or sinusoidally tapered waveguide can be analytically examined using infinite step discontinuities [12]. A $372 \mathrm{GHz}$ waveguide taper with a linear wall profile was designed for a rectangular-to-square (impedance ratio, $Z=2.00$ ) waveguide transition.

TABLE I: WAVEGUIDE SPLITTER BRANCH NUMBER AND POTENTIAL MODES.

\begin{tabular}{|c|c||c|}
\hline Branch No. & Converting Mode & Cavity TEmn Modes \\
\hline 2 & $\mathrm{TE}_{21}$ & $\mathrm{TE}_{21}, \mathrm{TE}_{41}, \mathrm{TE}_{61}, \ldots$ \\
3 & $\mathrm{TE}_{31}$ & $\mathrm{TE}_{31}, \mathrm{TE}_{61}, \mathrm{TE}_{91}, \ldots$ \\
4 & $\mathrm{TE}_{41}$ & $\mathrm{TE}_{41}, \mathrm{TE}_{81}, \mathrm{TE}_{12,1} \ldots$ \\
5 & $\mathrm{TE}_{51}$ & $\mathrm{TE}_{51}, \mathrm{TE}_{10,1}, \ldots$ \\
\hline \hline
\end{tabular}

A rectangular-to-square waveguide taper is shown in Fig. 1. The reflection coefficient $(\Gamma)$ of a rectangular waveguide taper supporting $\mathrm{TE}_{10}$ mode propagation can be defined as [11];

$$
\Gamma=\frac{\lambda_{g}}{L}\left[\frac{K_{0}^{2}+K_{1}^{2}}{64 \pi^{2}}-\frac{K_{0} K_{1}}{32 \pi^{2}} \cos (4 \pi l)\right]^{\frac{1}{2}}
$$

where $\lambda_{g}$ is the guide wavelength and $L$ is the tapering section length. $K_{0}, K_{l}$ and $l$ are given by

$$
\begin{aligned}
& K_{0}=\frac{\frac{\left(b_{1}-b_{0}\right)}{b_{0}}-\left(\left[\frac{\left(a_{1}-a_{0}\right)}{a_{0}}\right] /\left[1-\left(\frac{\lambda_{g}}{2 a_{0}}\right)^{2}\right]\right)}{\sqrt{1-\left(\frac{\lambda_{g}}{2 a_{0}}\right)^{2}}} \\
& K_{1}=\frac{\frac{\left(b_{1}-b_{0}\right)}{b_{1}}-\left(\left[\frac{\left(a_{1}-a_{0}\right)}{a_{1}}\right] /\left[1-\left(\frac{\lambda_{g}}{2 a_{1}}\right)^{2}\right]\right)}{\sqrt{1-\left(\frac{\lambda_{g}}{2 a_{1}}\right)^{2}}}
\end{aligned}
$$

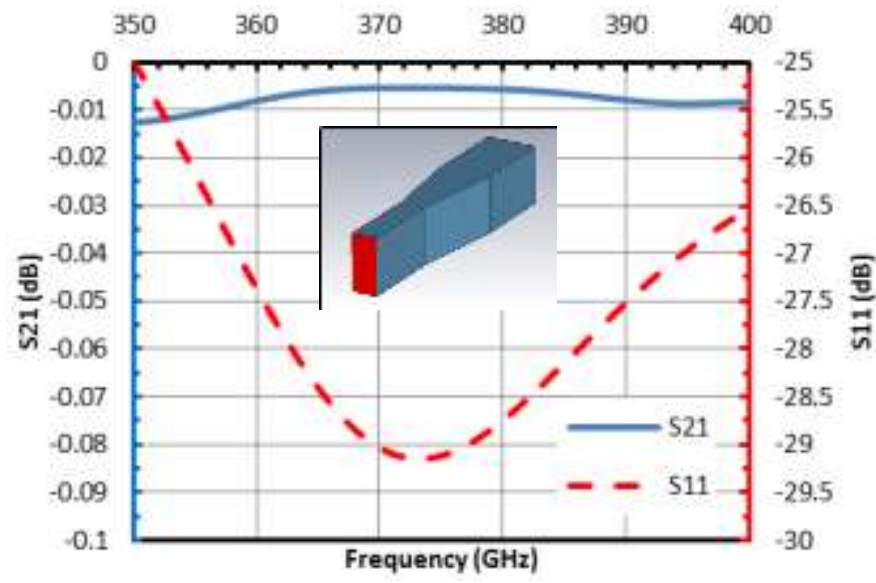

Fig. 2: Simulated scattering of a $372 \mathrm{GHz}$ rectangular-to-square waveguide taper.

$$
l=\frac{1}{\lambda_{g}} \int_{0}^{L} \sqrt{1-\left(\frac{\lambda_{g}}{2 a}\right)^{2}} d x
$$

respectively, where $a$ and $b$ denote the waveguide width and height with the subscripts 0 and 1 representing the initial and final magnitudes respectively. A variation of $a$ is only witnessed for a taper with an H-plane projection. For an E-plane rectangular-to-square taper where $a_{0}=a_{1}$ and $b_{1}=2 \cdot b_{0}$, equation (1) simplifies to

$$
\Gamma=\frac{\lambda_{g}}{16 \pi L}\left[5-4 \cos \left(\frac{4 \pi L}{\lambda_{g}}\right)\right]^{\frac{1}{2}}
$$

Analysis of equation (5) highlights a minimum reflection coefficient when $L \approx N \cdot \lambda_{g}$, where $N$ is a whole integer number. The guide wavelength at $372 \mathrm{GHz}$ is $\sim 1.10 \mathrm{~mm}$; therefore, the taper length is set at $\mathrm{N} \cdot 1.10 \mathrm{~mm}$. To minimise wall losses, the taper length should be minimised. Therefore, $N=1$.

A $372 \mathrm{GHz}$ impedance matching tapered waveguide was designed using CST Microwave Studio (CST-MS). The waveguide width $(a)$ was initially set at $0.60 \mathrm{~mm}$ with a waveguide width-to-height $(a: b)$ ratio of $1: 2$. The taper length (L) was set at $1.10 \mathrm{~mm}$. A parametric optimisation of $a$ was performed to minimise reflections at the rectangular input port (Port 1). The numerically simulated transmission and reflection of a $Z=2.00$ rectangular-to-square E-plane waveguide taper is shown in Fig. 2. The topology of the simulated waveguide taper is included in the inset. The waveguide width $(a)$ was optimised at $0.570 \mathrm{~mm}$, equating to $b_{0}=0.285 \mathrm{~mm}$ and $b_{1}=0.570 \mathrm{~mm}$, with the reflection at Port 1 less than $-27 \mathrm{~dB}$ over the desired operating bandwidth. Therefore, the low reflection component may be used at the incident port.

An E-plane waveguide bend is a fundamental component of waveguide engineering. A rotation of a waveguide about an angle, commonly between $15^{\circ}$ to $90^{\circ}$, creates a smooth-walled waveguide junction. The smooth E-plane transition can be designed to be compact (small $R_{I N}$ ) or broad (large $R_{I N}$ ) with small return losses. A smooth-walled $90^{\circ}$ E-plane waveguide bend with $a=0.570 \mathrm{~mm}$ and $b=0.285 \mathrm{~mm}$ was designed using CST-MS. The waveguide dimensions $a \times b$ coincide with the optimised tapered waveguide. The inner radius of the E-plane 
bend $\left(R_{I N}\right)$ was optimised to minimise reflections at the incident waveguide port. All values of $R_{I N}>0.05 \mathrm{~mm}$ will result in $\mathrm{S}_{11} \leq$ $-20 \mathrm{~dB}$; however, a radius increase up to $0.25 \mathrm{~mm}$ is shown to improve transmission. The radii selection is then dependant on minimising reflection at the centre of the operating bandwidth. The return loss from the E-plane bend is $<40 \mathrm{~dB}$ over the required $360-384 \mathrm{GHz}$ operating bandwidth.

The $372 \mathrm{GHz}$ HOM tapered-waveguide input coupler is designed using CST-MS to couple the $\mathrm{TE}_{10}{ }^{\mathrm{R}}$ mode to the $\mathrm{TE}_{61}{ }^{\mathrm{C}}$ mode using the waveguide transition and E-plane waveguide bend adjoined to a circular cavity of radius $(R)$. The average radius $\left(R_{0}\right)$ of the interaction cavity for the $372 \mathrm{GHz}$ gyro-TWA is $1.24 \mathrm{~mm}$; however, due to the enhanced coupling complexity to the $\mathrm{TE}_{61}$ mode, a small taper may be employed between the input coupler and the cavity to permit maximum coupling into the interaction cavity. Therefore, $R$ can be varied within 1.00 (cut-off radius of $\mathrm{TE}_{61}$ at $360 \mathrm{GHz}$ ) to $1.40 \mathrm{~mm}$ to support the desired $\mathrm{TE}_{61}$ mode and, hence, optimisation of $R$ may be carried out on the HOM input coupler. The beam tunnel is coupled to the rectangular input port from two discrete waveguide branches which consist of 3 E-plane bends adjoined to the tapered waveguide transition. The two waveguide branches evenly split the signal from the input port (Port 1), propagating the divided wave in the $\pm x$-direction before propagating the two synchronous signals into the beam cavity. The 2D progression of the mm-wave propagation is shown in Fig. 3.

Fig. 3 (left) highlights coupling to the $\mathrm{TE}_{61}{ }^{\mathrm{C}}$ mode for an optimised cavity radius $(R)$ of $1.03 \mathrm{~mm}$. The generated HOM will be able to freely propagate towards the cavity and the diode region unless Port 3, the port defined as being adjacent to the electron gun, is terminated. The average electron beam radius $\left(R_{B}\right)$ from the electron gun is $0.20 \mathrm{~mm}$ [13]; therefore, due to the HOM cavity a cut-off waveguide (Fig. 3 (right)) may be employed to prevent the propagation of incident radiation into the diode, without intercepting the electron beam. A parametric optimisation of $R_{C}$ is performed with a range of $(0.70-0.85)$ $\mathrm{mm}$ to cut-off all modes higher in frequency than the $\mathrm{TE}_{41}$ mode whilst ensuring a large beam tunnel diameter.

Fig. 4 highlights the $372 \mathrm{GHz} \mathrm{TE}_{10}{ }^{\mathrm{R}}-\mathrm{TE}_{61}{ }^{\mathrm{C}}$ tapered waveguide splitter input coupler operating between $359-385$ $\mathrm{GHz}$. The 3D E-field progression on the conductor walls is shown in the inset with port numbers defined. The bandwidth of the input coupler equates to $\sim 7 \%$. The cut-off waveguide reflector $\left(R_{C}=0.80 \mathrm{~mm}\right)$ is effective at preventing significant wave power from propagating towards the electron gun with a signal level of $<-15 \mathrm{~dB}$ for the dominant $\mathrm{TE}_{41}$ mode. The distance between the near edge of the rectangular waveguide branches and the waveguide reflector $\left(L_{1}\right)$ is $0.272 \mathrm{~mm}$. The coupler shows a high $\mathrm{TE}_{61}$ mode selectivity performance with low magnitude coupling to subsequent LOM's and HOM's. A gyrating electron beam will occupy $\sim 25 \%$ of the cut-off reflector cross-sectional area; hence, the potential of electron bombardment on the cavity boundaries is minimal and an increased beam diameter may be employed. Return loss at the input port (Port 1) is $<12 \mathrm{~dB}$ over the operating bandwidth of $360-384 \mathrm{GHz}$.

At $372 \mathrm{GHz}$, the skin depth of a mm-wave signal in a copper waveguide is $\sim 0.1 \mu \mathrm{m}$. Hence, the depth at which the signal propagates within the waveguide wall is comparable to, or smaller than, the surface roughness. Therefore, Ohmic losses

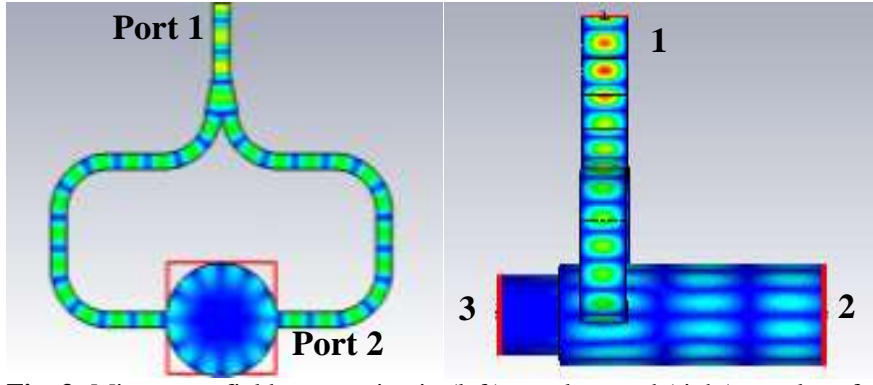

Fig. 3: Microwave field progression in (left) $x-z$ plane and (right) $y-z$ plane for tapered waveguide splitter input coupler.

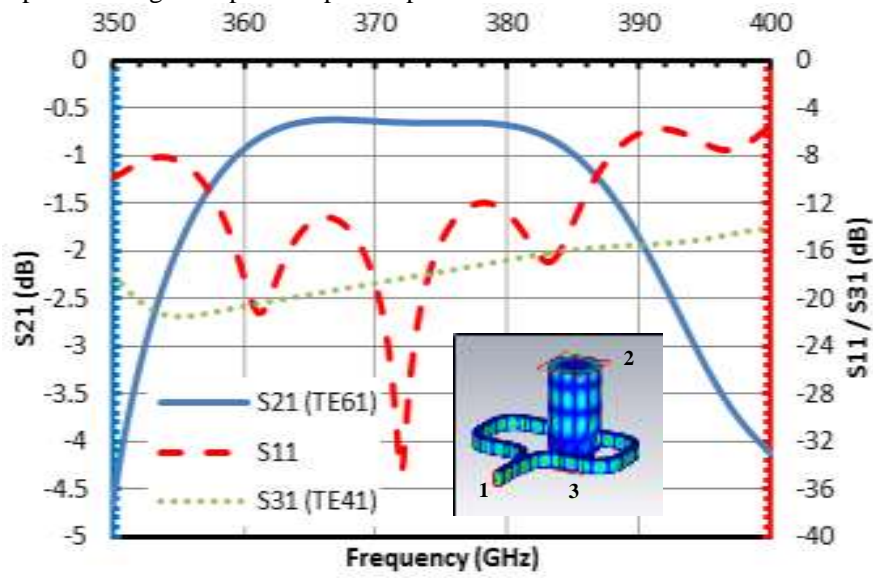

Fig. 4: Numerical scattering of a $\mathrm{TE}_{10}-\mathrm{TE}_{61}$ tapered waveguide splitter input coupler.

will increase with an uneven waveguide finish. With consideration of the surface effects on electrical conductivity, a reduction factor was applied to the copper conductivity $\left(0.5 \times 10^{7} \mathrm{Sm}^{-1}\right)$. The coupling strength at $372 \mathrm{GHz}$, numerically simulated with CST-MS, was shown to decrease by $0.91 \mathrm{~dB}$ with a coupling strength of $-0.77 \mathrm{~dB}$ for a perfect electrical conductor (PEC) background material and $-1.68 \mathrm{~dB}$ for a reduced conductivity copper background. A smooth surface finish $\left(R_{a}=2-3 \mu \mathrm{m}\right)$ must be achieved in manufacture to ensure a high $\mathrm{TE}_{61}$ coupling factor.

\section{TOLERANCE STUDY}

A tolerance study was performed on the $372 \mathrm{GHz}$ tapered waveguide input coupler to test the feasibility of the coupler performance with parametric discrepancies resulting from fabrication tolerances. Initially, the rectangular splitter waveguide is analysed. The parameters which govern the waveguide dimensions $\left(a, b, L\right.$ and $\left.R_{I N}\right)$ were varied from the optimum value to test the tolerance range over which the coupler may operate. A plot of waveguide width variation $(a)$ for fixed values of $b$ and $L$ is shown in Fig. 5.

The resultant change to the transmission coefficient for $a= \pm$ $20 \mu \mathrm{m}$ highlights an operational frequency shift of $\sim 0.5 \mathrm{GHz}$ for a $10 \mu \mathrm{m}$ variation. A frequency shift of $0.5 \mathrm{GHz}$ magnitude would not affect the coupler operation at $360-384 \mathrm{GHz}$; however, larger magnitude $a$ discrepancies would result in a frequency shift that was out with the desired bandwidth. The waveguide height $(b)$ was varied for fixed $a$ and $L$. The investigation highlighted minimal frequency or coupling strength response for $\Delta b= \pm 20 \mu \mathrm{m}$; therefore, the waveguide height has no strict tolerance requirement. The taper length $(L)$ is subsequently varied with no detrimental effects on coupling 


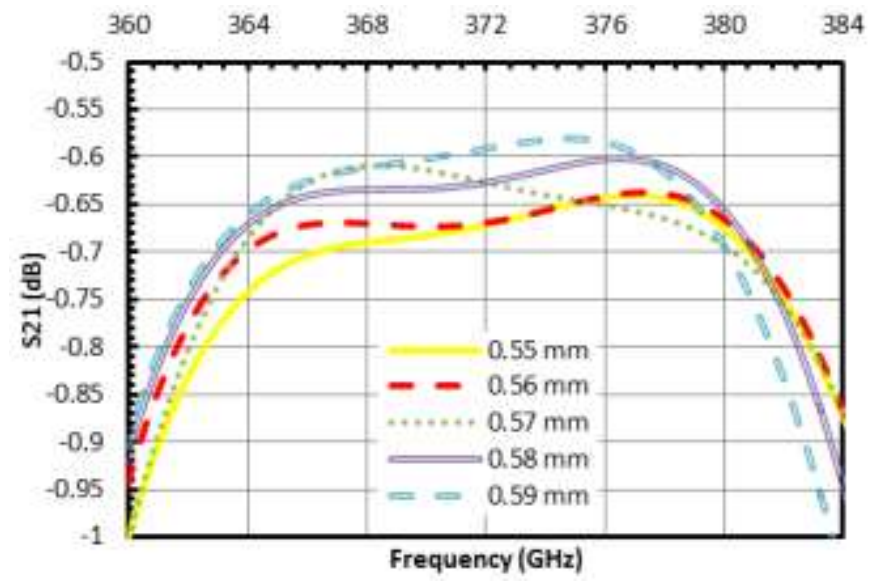

Fig. 5: Effect of $a$ parametric variation on $\mathrm{TE}_{61}$ input coupler transmission response.

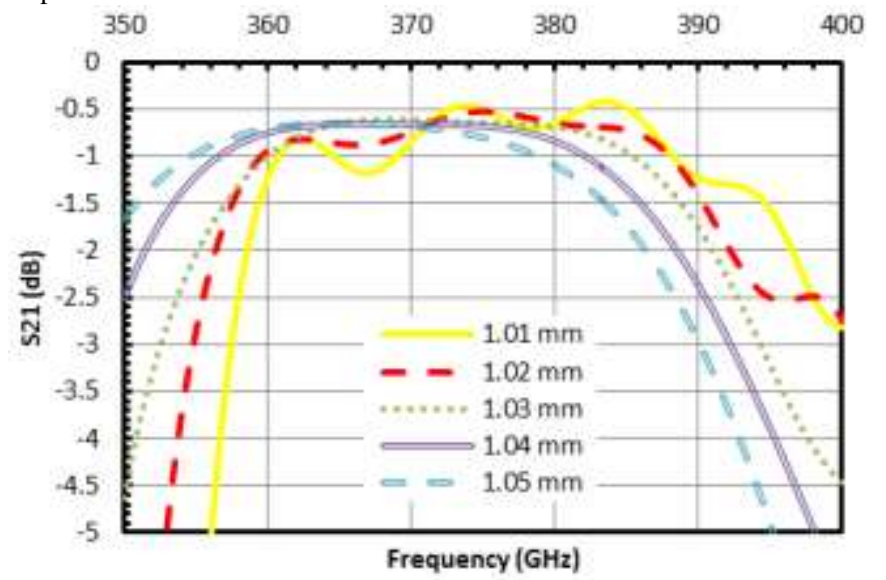

Fig. 6: $\mathrm{TE}_{61}$ Input coupler transmission with $R$ variations.

performance for $\Delta L \pm 25 \mu \mathrm{m}$. The waveguide bend radius $\left(R_{I N}\right)$ was investigated with optimum values of $a, b$ and $L$. For $\Delta R_{I N}$ values of $\pm 100 \mu \mathrm{m}$, no effect on coupling strength or frequency response was observed. However, an $R_{I N}$ increase will result in enhanced waveguide losses due to an increase in propagation length. The coupler performance as a result of rectangular waveguide discrepancies remains broadly unaffected.

An investigation into the coupled cavity is subsequently performed for $R$ and $L_{l}$. The cavity radius $(R)$ should be carefully manufactured to ensure a tight tolerance. Fig. 6 shows the effect of up to $\Delta R= \pm 20 \mu \mathrm{m}$ on the tapered input coupler performance. The effect of $R$ variations is shown to have a significant effect on the coupler centre frequency with a $10 \mu \mathrm{m}$ discrepancy in $R$ resulting in a $3 \mathrm{GHz}$ shift. Hence, the machining of $R$ must be very precise.

A review of the $L_{1}$ parameter was performed (Fig. 7). Alterations resulting in $25 \mu \mathrm{m}$ increases to $L_{l}$ are shown to decrease the centre frequency by $\sim 2 \mathrm{GHz}$. The discrepancies to $L_{l}$, which result in a phase mismatch between the incident and reflected wave at Port 3 , is shown to affect the coupler bandwidth and coupling strength with deviation from the optimum.

\section{INPUT COUPLER MANUFACTURE}

A W-band prototype $\mathrm{TE}_{61}$ tapered-waveguide input coupler was manufactured to confirm the simulated reflection results. The coupler was scaled to operate at $90-96 \mathrm{GHz}$ due to limitations on achievable tolerances using in-house

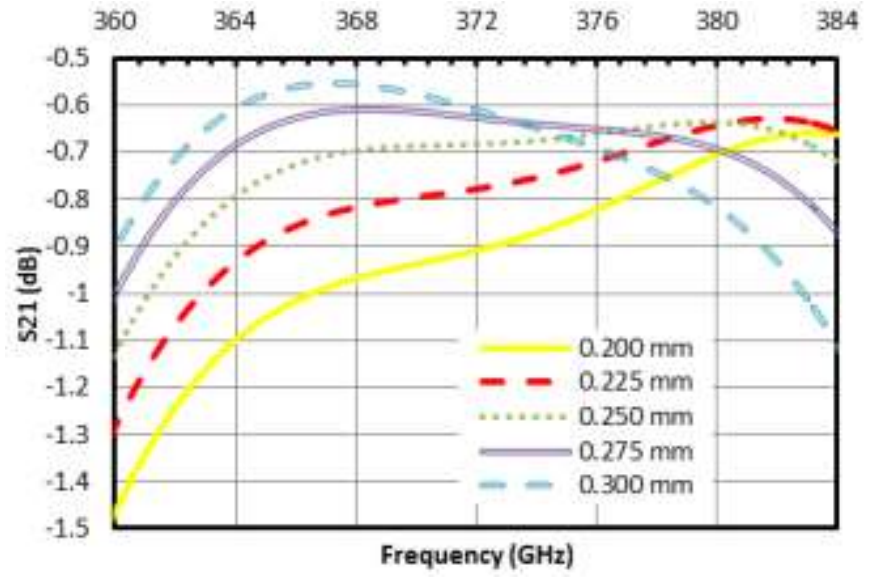

Fig. 7: Effect of $L_{l}$ variation on input coupler transmission response. manufacture. The circular coupled waveguide was scaled directly with a $4 \mathrm{x}$ dimension increase on the $372 \mathrm{GHz}$ design. The rectangular input waveguide was designed to maintain the aspect ratio used in the $372 \mathrm{GHz}$ design between standard WR2.2 ( $\left.a^{\prime} \times b^{\prime}\right)$ waveguide and $a_{0} \times b_{0}$. A tapered section from standard W-band waveguide to the optimised coupler waveguide is included in the scaled design.

The aluminium block was split into two discrete sections. A waveguide channel was manufactured into each split-block using a 3-axis Computer Numerically Controlled (CNC) milling technique. The tool path was machined into each split-block using a $0.60 \mathrm{~mm}$ end mill with half the waveguide channel width (1.27 mm depth) milled into each aluminium block. The length of the end mill cutter would prohibit the machining of the full waveguide width into a single block. The circular waveguide and waveguide reflector radii $(R=4.10 \mathrm{~mm}$ and $R_{c}=3.20 \mathrm{~mm}$ ) were subsequently drilled. A flange connection was machined into the split-block coupler to allow for direct attachment to the VNA. A series of threaded rods were used to join the split-block structure to prevent any loss of signal between the block faces. The manufactured waveguide channel is shown in Fig. 8.

\section{VECTOR Network ANALYSER TESTING}

TABLE II: WAVEGUIDE COUPLER DIMENSION COMPARISON

\begin{tabular}{|c||c||c||c||}
\hline \hline Parameter & $\begin{array}{c}\text { 372 GHz Design } \\
\text { (mm) }\end{array}$ & $\begin{array}{c}\text { 93 GHz Design } \\
\mathbf{( m m})\end{array}$ & $\begin{array}{c}\text { 93 GHz Actual } \\
\mathbf{( m m})\end{array}$ \\
\hline$R$ & 1.030 & 4.10 & 4.12 \\
$R_{C}$ & 0.800 & 3.20 & 3.45 \\
$a_{0}$ & 0.570 & 2.60 & 2.60 \\
$a^{\prime}$ & 0.560 & 2.54 & 2.60 \\
$b_{0}$ & 0.235 & 0.95 & 0.90 \\
$b^{\prime}$ & 0.280 & 1.27 & 1.23 \\
$b_{1}$ & 0.570 & 1.90 & 1.80 \\
$R_{I N}$ & 0.800 & 2.72 & 2.75 \\
$R_{O U T}$ & 1.085 & 3.67 & 3.65 \\
$L_{1}$ & 0.272 & 1.10 & 1.17 \\
\hline \hline
\end{tabular}

The experimental study was performed on an Anritsu 37000D Lightning series VNA with attached OML heads to enable frequency measurements in the range of $75-110 \mathrm{GHz}$. 


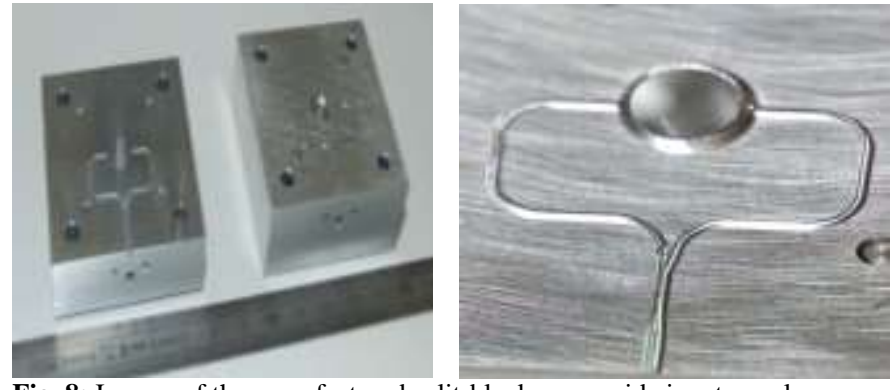

Fig. 8: Images of the manufactured split-block waveguide input coupler.

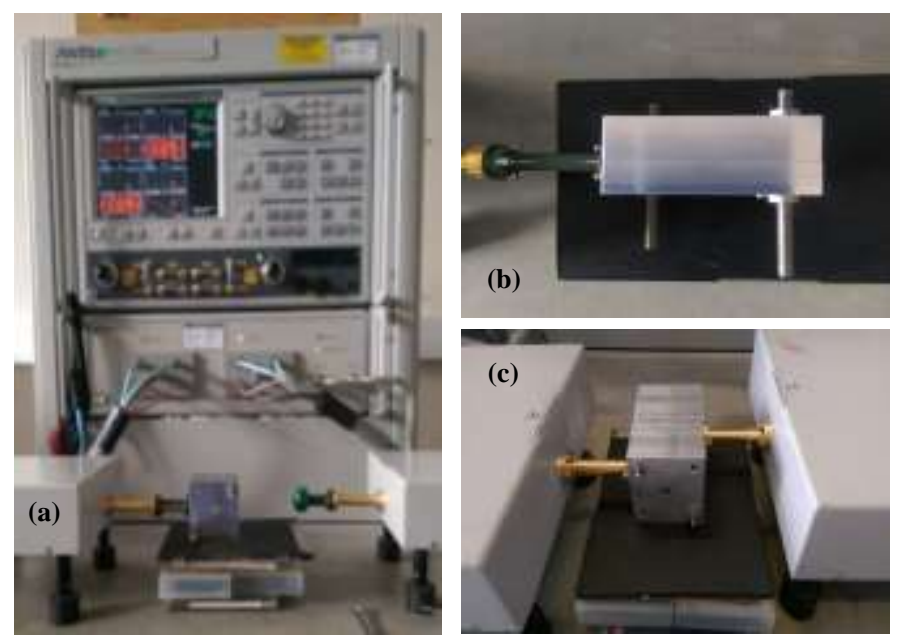

Fig. 9: VNA measurement setup with 2- and 4- block coupler.

Images of the coupler split-block attached to the VNA are shown in Fig. 9(a)-(b).

A comparison of the prototype $94 \mathrm{GHz}$ and $372 \mathrm{GHz}$ waveguide coupler dimensions is shown in Table II. A discrepancy between the designed and manufactured parameter sets was highlighted with precision measurement of the manufactured waveguide channel using a travelling microscope. The manufactured parameter set is included in Table II. A comparison of the $90-96 \mathrm{GHz}$ waveguide coupler numerical scattering with the designed and actual parameter set is shown in Fig. 10. The parametric discrepancies are shown to down shift the operating frequency range of the $\mathrm{W}$-band tapered waveguide coupler.

The HOM coupler numerical reflection for the designed and actual parameter set and VNA reflection results are shown in Fig. 11. Fig. 11 shows that the measured VNA reflection from the rectangular input port (Port 1) is in good agreement with CST-MS simulation results when open boundaries replace waveguide ports at Port 2 and Port 3 . The $S_{11}$ reflection at the input port is < - $10 \mathrm{~dB}$ between $90-96 \mathrm{GHz}$; therefore, $>90 \%$ of incident radiation is propagated into the coupled waveguide.

A reciprocal waveguide coupler was manufactured to allow for transmission and phase measurement. Therefore, the waveguide mode can be evaluated. The 4 block experimental setup is shown in Fig. 9(c). The transmission of two identical waveguide couplers back-to-back is shown in Fig. 12. The average transmission loss is $\sim 3 \mathrm{~dB}$ equating to an individual coupler loss of $-1.5 \mathrm{~dB}$. The circuit loss in an individual aluminium coupler block was measured at $-1 \mathrm{~dB}$; therefore, high mode conversion is exhibited by the HOM waveguide coupler.

To confirm the mode of conversion, an additional waveguide section of length $3.00 \mathrm{~mm}$ was manufactured and inserted

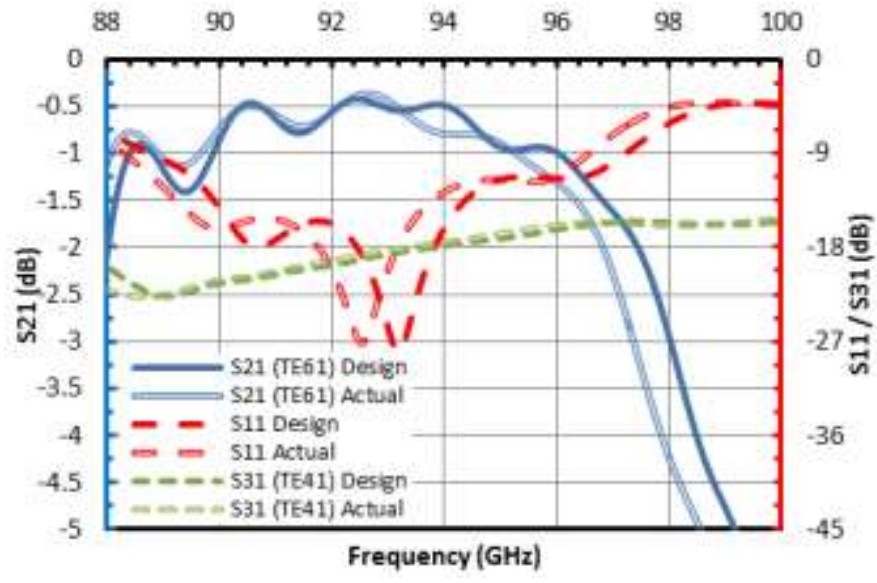

Fig. 10: Simulated transmission and reflection of W-band split block coupler for design and manufactured parameter sets.

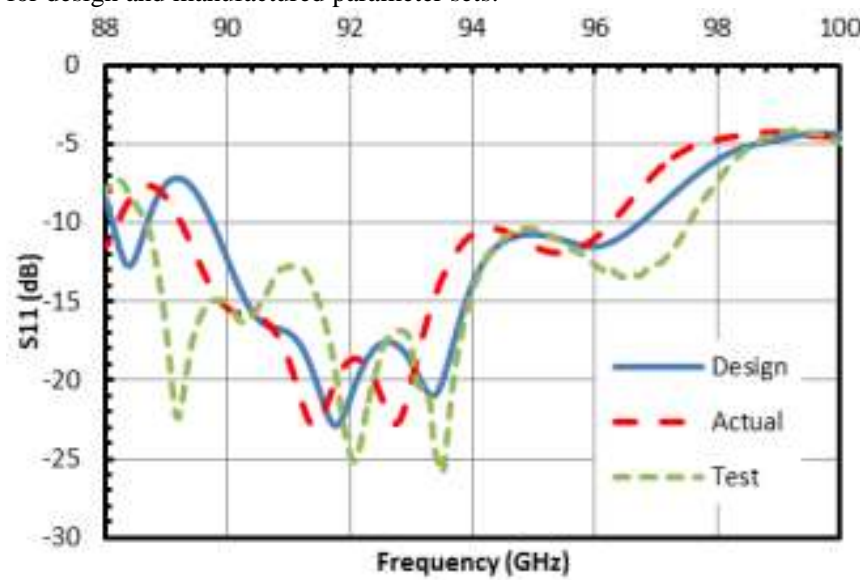

Fig. 11: Comparison of CST-MS and VNA coupler reflection results.

between the two couplers to measure the phase response. The measured phase was then compared with the theoretical phase response of the $\mathrm{TE}_{61}$ mode in a waveguide of radius $R=4.10$ $\mathrm{mm}$. The theoretical and measured phase is shown in Fig. 12. The phase measurement has close correlation to the theoretical $\mathrm{TE}_{61}$ phase at $88-98 \mathrm{GHz}$; therefore, the waveguide coupler is shown to have a high $\mathrm{TE}_{61}$ mode conversion efficiency. The insertion loss $(<10 \mathrm{~dB})$ and phase measurements suggest $>90 \%$ transmission to the $\mathrm{TE}_{61}$ mode.

\section{DISCUSSION AND CONCLUSION}

The design of a $372 \mathrm{GHz} \mathrm{TE}_{10}{ }^{\mathrm{R}}-\mathrm{TE}_{61}{ }^{\mathrm{C}}$ input coupler for a gyro-TWA has been presented. The design of the HOM input coupler focussed upon achieving a broadband coupling with a high transmission coefficient whilst ensuring the $\mathrm{TE}_{61}$ mode was dominant within the coupled cavity. The tapered waveguide input coupler was designed with low loss rectangular waveguide components. The tapered waveguide was shown to have a return loss of $<27 \mathrm{~dB}$. The incident split signal was coupled into a circular waveguide through smooth walled E-plane waveguide bends. The waveguide bends had a reflection coefficient of $<-40 \mathrm{~dB}$ over the $360-384 \mathrm{GHz}$ operating bandwidth. The tapered waveguide input coupler achieved an operating bandwidth of $7 \%(359 \mathrm{GHz}-385 \mathrm{GHz})$.

A tolerance study of the $\mathrm{TE}_{10} \mathrm{R}-\mathrm{TE}_{61}{ }^{\mathrm{C}}$ tapered waveguide input coupler was performed to analyse the effect of manufacturing discrepancies on the coupler performance. The coupler was shown to be stable with manufacturing 


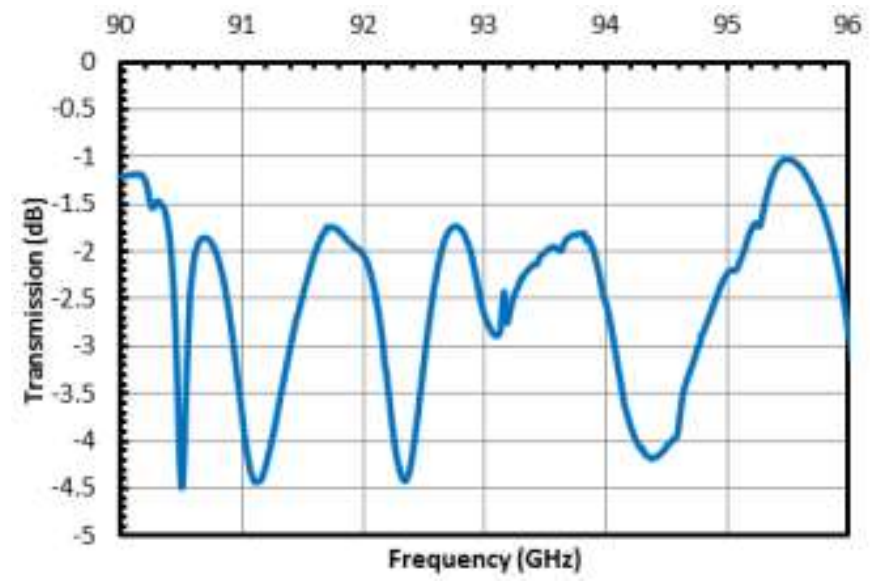

Fig. 12: Transmission of W-band HOM input coupler.

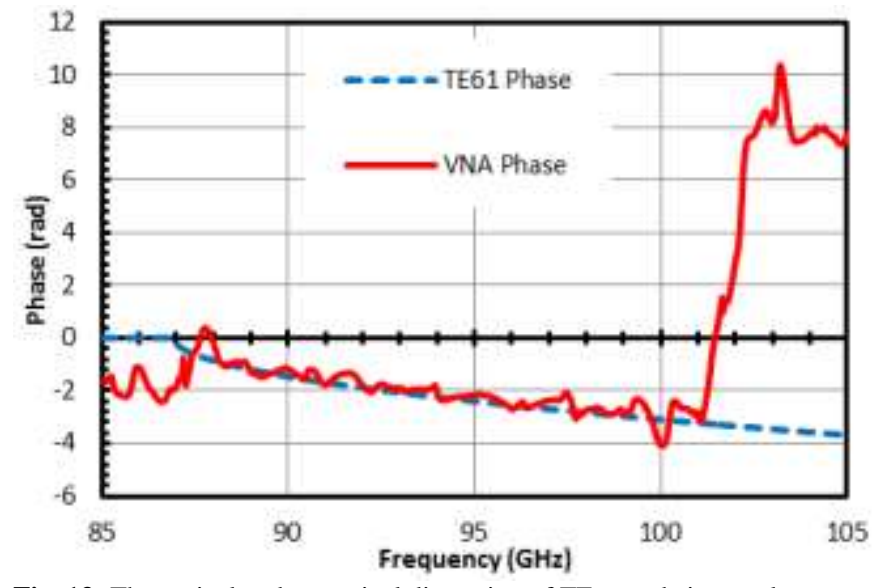

Fig. 13: Theoretical and numerical dispersion of $\mathrm{TE}_{61}$ mode in coupler. discrepancies associated to the rectangular splitter waveguide. A maximum coupling change of $0.1 \mathrm{~dB}$ was observed for 20 $\mu \mathrm{m}$ variations of $a$ corresponding to a frequency shift of $\sim 0.5$ GHz. An investigation into the coupled cylindrical beam tunnel, however, highlighted a frequency and coupling strength sensitivity with $R$ and $L_{l}$ variations. For $\Delta R= \pm 10 \mu \mathrm{m}$, a $3 \mathrm{GHz}$ centre frequency shift was observed and for $\Delta L_{l}= \pm 25 \mu \mathrm{m}$ the centre frequency was shifted by $\sim 2 \mathrm{GHz}$. A decrease in coupling strength was also associated with $R$ and $L_{l}$ discrepancies (Fig. 6 and Fig. 7). Therefore, to ensure high coupling over the desired $360-384 \mathrm{GHz}$ bandwidth the beam tunnel must be accurately manufactured with strict tolerances applied.

A W-band $\mathrm{TE}_{61}$ prototype coupler was manufactured to allow for VNA testing of the tapered waveguide input coupler. A CNC milling technique was used to machine a waveguide channel designed to operate at $90-96 \mathrm{GHz}$. The waveguide dimensions were scaled from the optimised $360-384 \mathrm{GHz}$ $\mathrm{TE}_{10}{ }^{\mathrm{R}}-\mathrm{TE}_{61}{ }^{\mathrm{C}}$ tapered waveguide input coupler. The 2-piece aluminium split block coupler design allowed for VNA testing of the coupler reflection. The measured $S_{11}$ reflection from the input rectangular port (Port 1) of the coupler structure was shown to be $<-10 \mathrm{~dB}$ between $90-96 \mathrm{GHz}$. The VNA measurement agreed well with simulated data, suggesting $90 \%$ of incident radiation is transmitted into the circular coupled waveguide. A transmission and phase measurement with a reciprocal input coupler was presented. The phase difference analysis permitted the determination of the $\mathrm{TE}_{61}$ conversion into the coupled waveguide with the measurement shown to be in good agreement with theoretical $\mathrm{TE}_{61}$ phase over the operating bandwidth.

The manufacturing technique outlined for the W-band HOM input coupler may be used in the manufacture of a $372 \mathrm{GHz}$ waveguide coupler; however, state-of-the-art micro-milling technology must be employed. High precision laboratories [14] have the capability to machine waveguide channels of $0.1 \mathrm{~mm}$ width with a positional accuracy of $\pm 0.3 \mu \mathrm{m}$ using 5 -axis micro-milling machines and an appropriate end-mill tool piece. A surface finish with $R_{a}=\sim 2 \mu \mathrm{m}$ would ensure that Ohmic losses would be minimised. Therefore, the design of a HOM power splitting waveguide coupler with waveguide sub-mm $a \mathrm{x}$ $b$ cross-section $(0.57 \times 0.24 \mathrm{~mm})$ is realistic.

\section{REFERENCES}

1. K. R. Chu, "The electron cyclotron maser," Rev. Mod. Phys. vol. 76, no. 2, pp. 489-540, 2004.

2. S. Coda et al., "Electron cyclotron current drive and suprathermal electron dynamics in the TCV tokamak," Nucl. Fusion, vol. 43, no. 11, pp. 1361 1370, 2003.

3. M. G. Czerwinski and J. M Usoff, "Development of the Haystack Ultrawideband Satellite Imaging Radar," Linc. Lab. J., vol. 21, no. 1, pp. $28-44,2014$

4. E. A. Nanni et al., "THz Dynamic Nuclear Polarization NMR," IEEE THz Sci. Techn., vol. 1, no. 1, pp. 145 - 163, 2011.

5. L. Zhang et al., "Multi-mode coupling wave theory for helically corrugated waveguide," IEEE Trans. Microw. Theory Techn. vol. 60, pp. 1-7, 2012.

6. L. Zhang et al., "Design and Measurement of a Broadband Sidewall Coupler for a W-Band Gyro-TWA", IEEE Trans. Microw. Theory Tech., vol. 63, pp. 3183-3190, 2015.

7. V. L. Bratman et al., 'High-gain wide-band gyro-travelling wave amplifier with a helically corrugated waveguide', Phys. Rev. Lett., vol. 84, pp. 2746-2749, 2000.

8. W. He et al., "High power wideband gyrotron backward wave oscillator operating towards the terahertz region," Phys. Rev. Lett. vol. 110, pp. 165101, 2013.

9. C. F. Yu and T. H. Chang, "High-performance circular TE01-mode converter," IEEE Transactions on Microwave Theory and Techniques, vol. 53, no. 12, pp. 3794-3798, 2005.

10.K. Matsumaru, "Reflection coefficient of E-plane tapered waveguides," IRE Trans. Microw. Theory Techn. vol. 6, no. 2, pp. 143 - 149, 1958.

11.R. C. Johnson, "Design of linear double tapers in rectangular waveguide," IRE Trans. Microw. Theory Techn. vol. 7, no. 3, pp. $374-378,1959$.

12.R. A. Waldron, "Theory of reflections in a tapered waveguide," $J$. I. Electron. Rad. Eng. vol. 32, no. 4, pp. 245 - 254, 1966.

13.J. R. Garner et al., "Design Study of a Fundamental Mode Input Coupler for a 372-GHz Gyro-TWA I: Rectangular-to-Circular Coupling Methods," IEEE Trans. Electron Devices, vol. 63, no. 1, pp. 497 - 503, 2016.

14.Science and Technology Facilities Council, "CNC Nano-machining," www.ralspace.stfc.ac.uk, 2016. 